\title{
Ferrite HOM Absorber for the RHIC ERL
}

\author{
H. Hahn, E.M. Choi, L. Hammons
}

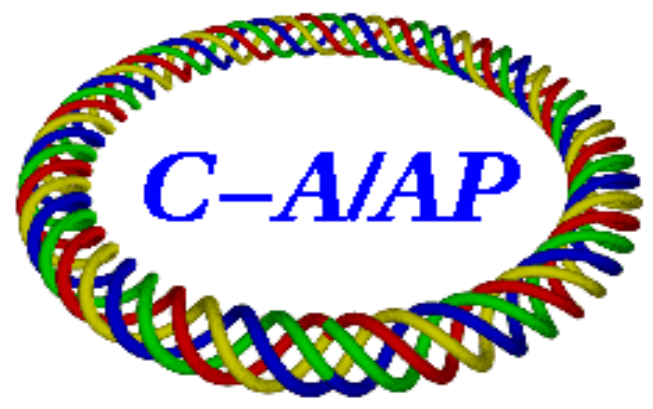

Collider-Accelerator Department Brookhaven National Laboratory Upton, NY 11973

Notice: This document has been authorized by employees of Brookhaven Science Associates, LLC under Contract No. DE-AC02-98CH10886 with the U.S. Department of Energy. The United States Government retains a nonexclusive, paid-up, irrevocable, world-wide license to publish or reproduce the published form of this document, or allow others to do so, for United States Government purposes. 


\title{
Ferrite HOM absorber for the RHIC ERL
}

\author{
H. Hahn, E. M. Choi, and L. Hammons \\ Collider-Accelerator Department, Brookhaven National Laboratory \\ Upton, New York, 11973-5000
}

\begin{abstract}
A superconducting Energy Recovery Linac is under construction at Brookhaven National Laboratory to serve as test bed for RHIC upgrades. The damping of higher-order modes in the superconducting five-cell cavity for the Energy-Recovery linac at RHIC is performed exclusively by two ferrite absorbers. The ferrite properties have been measured in ferrite-loaded pill box cavities resulting in the permeability values given by a first-order Debye model for the tiled absorber structure and an equivalent permeability value for computer simulations with solid ring dampers. Measured and simulated results for the higher-order modes in the prototype copper cavity are discussed. First roomtemperature measurements of the finished niobium cavity are presented which confirm the effective damping of higher-order modes in the ERL by the ferrite absorbers.
\end{abstract}

\section{INTRODUCTION}

A superconducting Energy Recovery Linac (ERL) is under construction at Brookhaven National Laboratory (BNL) to investigate concepts for high-energy electron cooling in the Relativistic Heavy Ion Collider (RHIC) and for an envisioned electron-ion collider [1]. The ERL test facility is based on a niobium superconducting (SC) $703.75 \mathrm{MHz}$ "Electron Cooling Xperimental" (ECX) 5-cell cavity capable of accelerating electrons to $20 \mathrm{MeV}$ and an ampere-class SC 2.5 MV electron gun [2]. Damping higher order modes (HOM) in the SC ERL cavity is one of several challenges and, following the techniques developed at Cornell [3,4] and KEKB [5], has been addressed exclusively by means of ferrite absorbers. The cavity design [6] intentionally provided enlarged beam tube apertures to allow propagation of HOMs towards the absorbers located at room temperature. The 5-cell cavity was designed by BNL, the niobium structures and cryomodules were fabricated by AES on Long Island [7], the ferrite absorbers were fabricated according to Cornell-print by ACCEL in Germany [8] and the cavity was processed at the Thomas Jefferson National Accelerator Facility. The ERL cavity has been delivered and cold emission tests are scheduled for later this year. The RHIC ERL cavity string assembled in its helium vessel with the thermal isolation sections and the ferrite absorber is shown in Fig.1.

Designing superconducting cavities and predicting their performance can be done with great accuracy via the available computer programs. In contrast, adding lossy ferrite in large structures with complicated geometry eliminates the use of many open (free) simulation programs, and presents a considerable challenge in terms of the computer time required even in commercial programs [9]. Nevertheless, the use of a simulation program is productive and the CST Microwave Studio (MWS) [10] was extensively used 
for the ERL in the design phase and for the interpretation of prototype test results. In this paper, the study of the damping of the dominant lower-frequency dipole and monopole modes in the available prototype 5-cell copper cavity achieved with the model ferrite absorber is presented. The completion of the ERL cavity, even before cold tests, then provided a preliminary confirmation of the HOM damping by the ferrite absorber.

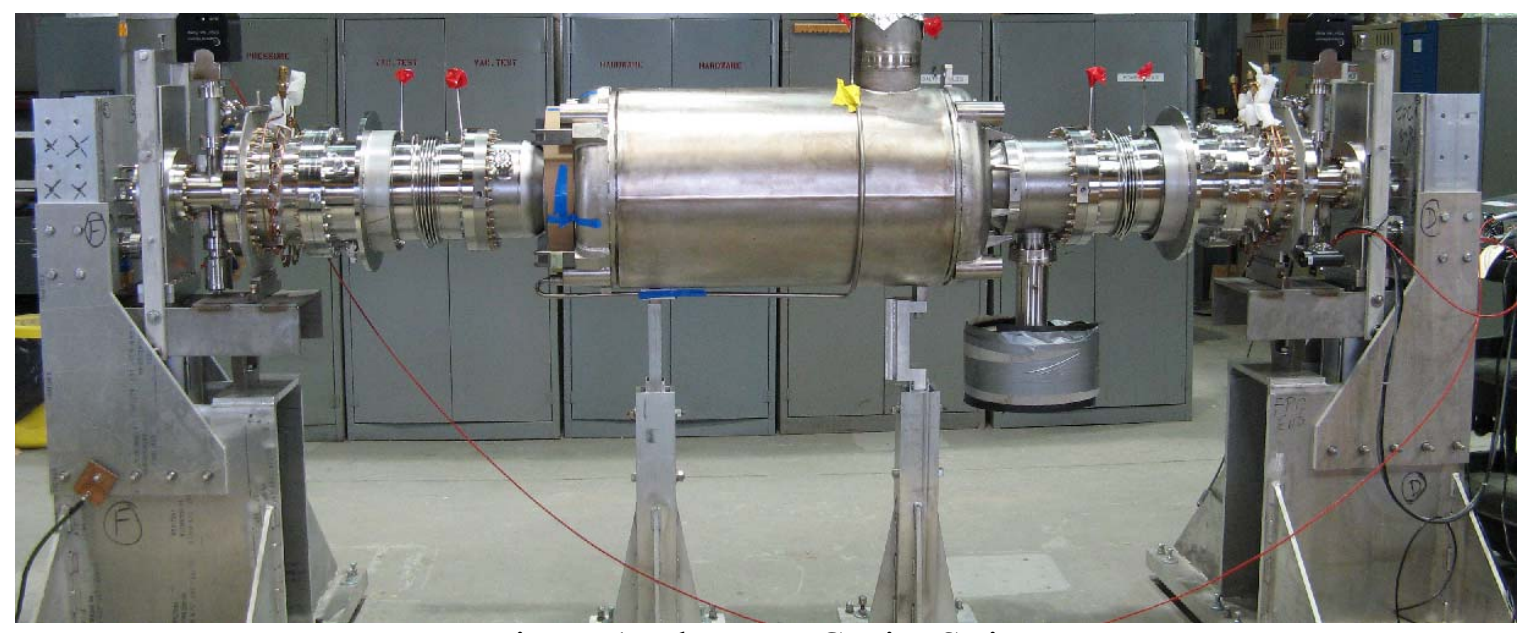

Figure 1. The ERL Cavity String

Investigating the freestanding HOM absorber properties was a central objective in this work. The approach taken consisted of transforming the model absorber into a ferrite loaded pillbox for standard scattering coefficient measurements and the interpretation of experimental data with simulation in the MWS from which the ferrite permeability was extracted. The absorber and its model is constructed as an assembly of many small ferrite tiles with interspersed gaps and exposed metallic stripes which, in the simulation program, requires large mesh sizes resulting in prohibitive running times. Therefore, a concomitant objective for the present study was finding portable absorber properties via parameters which describe the damping globally without the need of considering the tiled structure. Portability was obtained in the form of a ferrite cylinder with equivalent permeability and permittivity for use in simulation programs.

A large quantity of experimental data was collected over a period of about two years and preliminary results have been reported elsewhere [11-13]. For this paper, all results were reviewed and the more definite data are presented. In Section II, the prototype absorber is described and the MWS simulated accelerator properties of the fundamental mode in the prototype copper cavity are given. Forward scattering coefficient measurements of the cavity, performed with and without the absorber, over the interesting frequency range from 0.5 to $3 \mathrm{GHz}$ show the desired HOM damping. However, extracting quantitative permeability values from these global measurements is difficult and required the more specific studies discussed in the next sections.

A more direct way of measuring the ferrite damping properties was found by placing shorting plates at either end of a fully detailed model with ferrite tiles to form a low-Q "pillbox" cavity for standard network analyzer measurements. The Section III treats the case of a very short (VS) cavity, determined by the $6.5 \mathrm{~cm}$ length of a single ferrite tile, 
where the lowest resonance frequencies, TM010 and TM020, remain uncoupled. The TM010 resonance curve with frequency and Q-value can be obtained from S21 scattering coefficient measurements with sufficient accuracy. Extracting portable ferrite permeability parameters at this frequency is done by searching for a match with the MWS eigenvalue solver for varied complex- $\mu$ values. The ferrite permeability data over the full frequency range represented by the first order Debye model are obtained by matching of measured S21 transmission coefficients in the VS cavity with the simulation results from the MWS frequency domain solver.

An analysis of the data from VS cavity, taking into account the tiled structure, would require the development of a framework involving theoretical field analysis as well as the use of computer codes. A rigorous analytical treatment of the absorber with its ferrite tiles is lengthy and of limited value. In the Appendix it is replaced by a rigorous analysis of the skin effect in flat ferrite plates and then by treating the skin effect in a wave guide lined with a circularly and longitudinally uniform ferrite cylinder. The results justify the use of circular cylinders with lossy material instead of the tiled geometry in simulation programs requiring symmetry. The concept of approximating the tiled absorber with solid circular rings is discussed in Section III using the data TM010 resonance in the VS "pillbox" cavity.

Placing shorting plates at the ends of the two-tile long prototype absorber would lead to overlapping resonances and does not allow a simple interpretation of the network analyzer measurements. As demonstration of data portability and the resulting simplification from this approximation, a "double" (DBL) cavity was formed by combining the prototype absorber with a beam tube section of equal length, and the results are presented in Section IV. As a further test of the solid ring approximation, the damped HOMs of the copper cavity with the prototype absorber attached are simulated and compared with the experimental results in Section V. Finally, room temperature measurements performed on the ERL string and the effort at HOM identification are discussed in Section VI.

\section{5-CELL CU CAVITY AND FERRITE HOM ABSORBER}

Prior to the final design of the $\mathrm{Nb}$ cavity, methods of avoiding trapped HOMs were investigated and a 5-cell $\mathrm{Cu}$ cavity was fabricated to study HOM damping. Adding the prototype absorber to the $\mathrm{Cu}$ cavity and performing room temperature measurements provided data for several critical HOMs and confirmed the suitability of the exclusive use of ferrite absorbers [14]. The major objective of the subsequent R\&D work presented here was to determine the ferrite properties in a form that is portable into the MWS for the simulation of the ERL and other future cavities. However, the $\mathrm{Cu}$ cavity measurements did not provide the ferrite properties of the absorber in the detailed form required for the simulations and this task had to be addressed in compact ferrite loaded pill-box cavities as discussed in the subsequent sections.

The ERL cavity center cell is fabricated with a $41.874 \mathrm{~cm}$ equator, a $17.023 \mathrm{~cm}$ iris diameter, and a cell length of $21.331 \mathrm{~cm}$ at room temperature for cold operation at 703.75 
MHz. The end cell is adjusted for field flatness and the cavity is connected to the HOM absorber by nominally $24 \mathrm{~cm}$ diameter beam tubes. The production HOM absorber for the superconducting ERL cavity is a cylindrical spool with ferrite tiles soldered to $10 \mathrm{~W} 3$ Elkonite plates at the wall [15], similar to the prototype model shown in Fig. 2 . The unit has 18 plates in the $25 \mathrm{~cm}$ diameter spool, each with two tiles of $2 \times 1.5 \times 0.125 \mathrm{in}$. dimensions. Completing the description of the absorber requires the knowledge of the microwave ferrite properties.

The use of microwave absorbing ceramic and ferrite materials is an important topic to various areas of technology and has previously been considered for the damping of HOMs at accelerator laboratories [16]. Measurements of the microwave properties of ferrite materials have been performed and reported by several researchers: Hartung et al. [17] used coaxial transmission line techniques, whereas Tajima et al. [18] combined a similar pulse method with direct network analyzer measurements of ferrite-damped cavity resonances. The absorbing material used here for the ERL absorber is a C48 ferrite [19] the properties of which are available from the work by Mouris and Hutcheon (MH) [20] and some from Shemelin et al. [21]. The MH data for complex microwave permeability and permittivity data of the $\mathrm{C} 48$ ferrite was obtained from measurements of small pellets, $3.5 \mathrm{~mm}$ in diameter and $3.175 \mathrm{~mm}$ thick at frequencies from 915 to 2800 $\mathrm{MHz}$, covering the frequency range of interest to the present study. Although relevant, any effect due to the geometrical difference in the tiled absorber structure had to be determined on the prototype model for the purpose of this study.

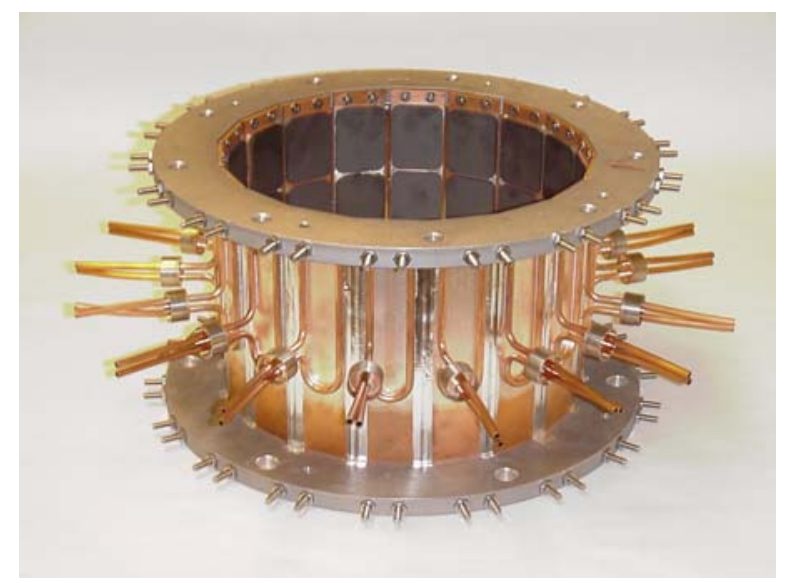

Figure 2. HOM ferrite absorber prototype

The damping properties of the ferrite were studied globally by determining the frequencies and quality factors of the HOMs in the Cu cavity with and without the absorber via forward scattering coefficient measurements with a network analyzer. For this, the cavity is excited by an input probe centered on the shorting disk on the left side in the picture, shown in Fig. 3. The output signal is taken either with a probe on the shorting disk on the right end or at the cavity side with the pick-up (PU) probe intended for RF operation of the $\mathrm{Nb}$ cavity. The pick-up probe is sensitive to monopole as well as dipole modes, and, due to its close-by location, sees all cavity modes. The axially located probe at the end strongly favors monopole modes but is separated from the cavity by the beam tube with its $\sim 24 \mathrm{~cm}$ diameter. The beam tube limits wave propagation of the 
monopole modes to the end probe below $\sim 957 \mathrm{MHz}$, but allows propagation to the $\mathrm{PU}$ probe of hybrid dipole modes at frequencies above $\sim 733 \mathrm{MHz}$.

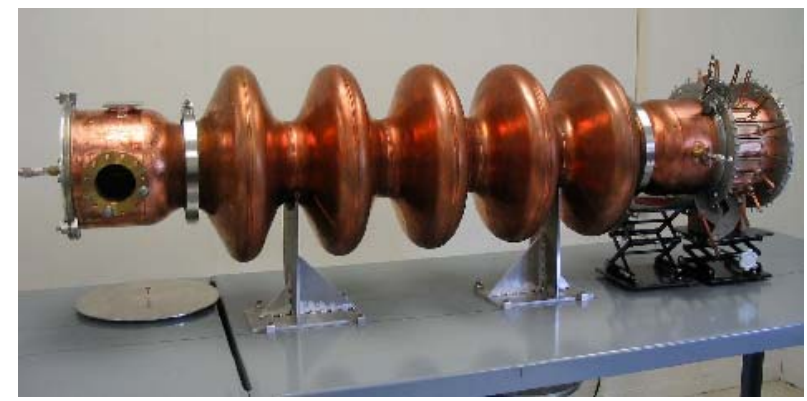

Figure 3. The 5-Cell Cu Cavity with prototype ferrite HOM absorber

For the measurements, the signal level is maximized by adjusting the input probe to achieve critical coupling for the fundamental cavity resonance at $703 \mathrm{MHz}$. The S21 transmission coefficients from input to the end and the PU probes are shown in Fig. 4. In order to maintain the electrical length and node positions of the standing waves, the ferrite absorber is replaced by a beam tube of equal length for the state without ferrite. The output signal at the cavity end, yellow, shows 1) clearly the TM01 wave suppression by the beam tube below cut off, 2) the strong damping by the absorber, black, and 3) the relative weak signal on axis of dipole modes, red. The $\mathrm{Cu}$ cavity measurements provide a global confirmation of the effective damping of the HOM by the ferrite absorber. A more detailed study will be presented in the subsequent sections.

S21 in Cavity+ FRT

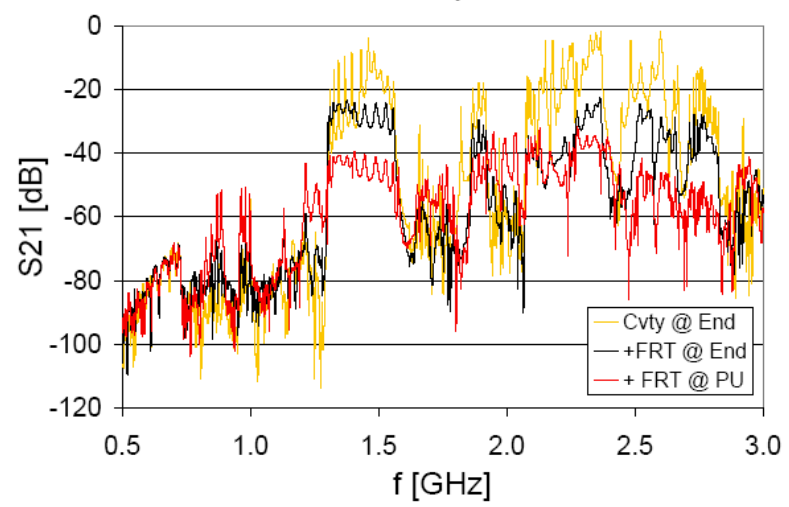

Figure 4. S21 in Cu Cavity without (yellow) and with (black and red) ferrite absorber

In preparation for the discussion of HOMs, a MWS model of the bare ERL cavity without absorber or beam tubes was constructed based on the detailed AES fabrication drawings. The model was tested against the fundamental modes in the copper cavity, which due to the TM01 cutoff frequency of the beam tube are not affected by the presence of the absorber. The measured frequencies and the MWS simulation results for the fundamental resonance group in the 5-cell ERL cavity are given in Table I. To serve as reference, the $R / Q$ "accelerator" values and the quality factors for the $\mathrm{Cu}$ cavity with $\sigma=5.8 \times 10^{7} / \Omega \mathrm{m}$ are also listed. The modes, with $m$ the mode number, agree with the expected frequency dependence [22] 


$$
\left(\frac{f_{m}}{f_{0}}\right)^{2}=1+2 \kappa\left[1-\cos \left(\frac{m}{5} \pi\right)\right]
$$

by taking the TM01 cell frequency $f_{0}=681.3 \mathrm{MHz}$ and the coupling coefficient $\kappa=0.0154$.

Table I. ERL cavity fundamental modes

\begin{tabular}{|c|c|c|c|c|}
\hline$m \pi / 5$ & $f_{\text {Data }}[\mathrm{MHz}]$ & $f_{\mathrm{MWS}}[\mathrm{MHz}]$ & $R / Q[\Omega]$ & $Q_{\mathrm{Cu}}$ \\
\hline 1 & 683.8 & 683.3 & 0.00 & 21,600 \\
\hline 2 & 688.9 & 688.3 & 0.05 & 22,200 \\
\hline 3 & 695.6 & 694.7 & 0.00 & 23,000 \\
\hline 4 & 700.9 & 699.9 & 0.20 & 23,700 \\
\hline 5 & 703.0 & 702.0 & 397.76 & 22,100 \\
\hline
\end{tabular}

\section{VERY SHORT PILL-BOX CAVITY}

The primary method of finding the $\mathrm{C} 48$ ferrite properties used in the HOM absorber consisted in assembling a ferrite-lined metal ring and placing shorting plates at either end thereby transforming it into a low- $Q$ pill-box cavity for standard network analyzer measurements. Interpretation of the $Q$ and S21 transmission measurements with MWS simulations leads to ferrite properties portable to the ERL cavity. Simulations over the full frequency from 0.5 to $3 \mathrm{GHz}$ with varied ferrite parameters in the search of a match with the S21 measurements depend on the dispersive model taken and can become excessively time consuming. Using the MWS eigenvalue solver requires cavities in which the resonances are clearly measurable, such as in the "double-cavity" discussed later, but precludes the $18.5 \mathrm{~cm}$ long prototype model with its overlapping resonances.

For the basic ferrite measurements, a very short (VS) $6.5 \mathrm{~cm}$ long pill-box model was assembled with the express goal to study the TM010 resonance at $\sim 800 \mathrm{MHz}$ which can be clearly identified and analyzed with the MWS simulation program. The MWS model for the VS cavity is shown in Fig. 5. The available number of ferrite tiles was limited to 16 , forcing the gap at the top and bottom, with the consequence of underestimating the impact on the damping properties. Nevertheless, matching the measured with the simulated results yields the ferrite parameters at this frequency. The ferrite parameters so obtained can then be tested over the full frequency range.

The measured forward scattering coefficients represent the basis for the analysis. The $\mathrm{S} 21$ coefficient is written in the conventional terminology as

$$
S_{21}=\frac{2 Q_{L}}{\sqrt{Q_{X i} Q_{X o}}\left(1+j Q_{L} \Delta\right)}
$$

with $\Delta=\left(\frac{\omega}{\omega_{0}}-\frac{\omega_{0}}{\omega}\right)$ and $\frac{1}{Q_{L}}=\frac{1}{Q_{0}}+\frac{1}{Q_{X i}}+\frac{1}{Q_{X o}}$

where $Q_{0}, Q_{X i}, Q_{X o}$ are the unloaded and external Q-factors respectively. Although these equations are rigorous, their practical use assumes only a field "perturbation" and thus a 
a frequency independent external $Q_{X}$, which is achieved by selecting weakly coupled, equal input and output probes. The MWS program does not provide the option of fixing the mesh structure under material changes. In order to ensure the correct interpretation of the MWS simulation results, the model and its mesh properties must be tested against measurements of a reference cavity at frequencies well below the first resonance, $\omega \ll \omega_{0}$, where the S21 is the same for the ferrite loaded and reference cavity,

$$
S_{21} \approx j \frac{\omega}{\omega_{0}} \frac{2}{\sqrt{Q_{X i} Q_{X o}}} .
$$

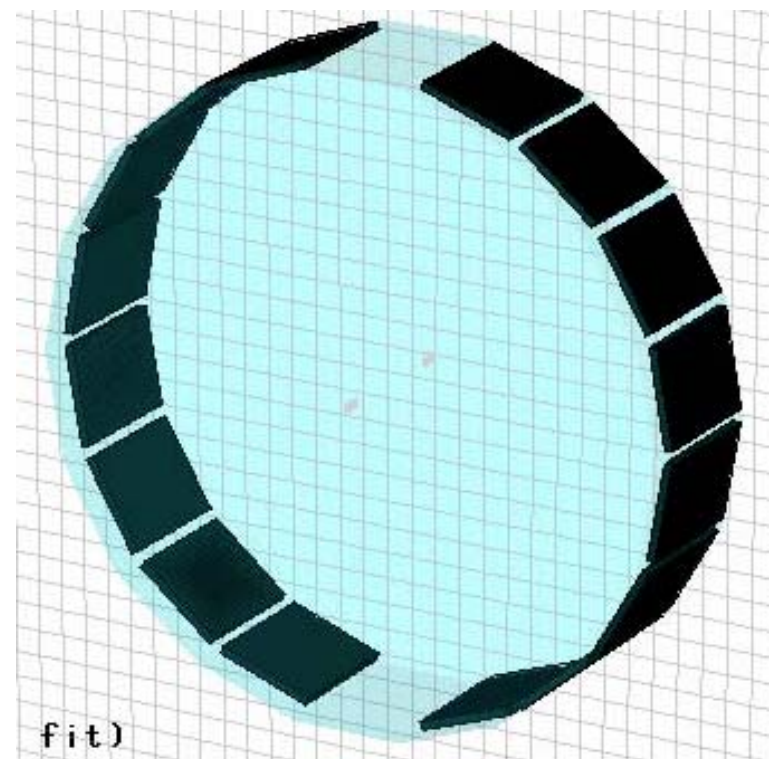

Figure 5. Fully detailed MWS model of the VS cavity

The data for the ferrite loaded VS cavity and a reference cavity of equal dimensions are shown in Fig. 6. The resonances in the reference cavity are TM010, TM020, and TM011 at $0.925,2.12$, and $2.49 \mathrm{GHz}$ respectively. The TM010 resonance in the loaded cavity retains its character and is a good source for the ferrite parameters. A more flexible approach of matching the resonance is based on finding the S21 curves with the frequency domain solver over a somewhat broader range.

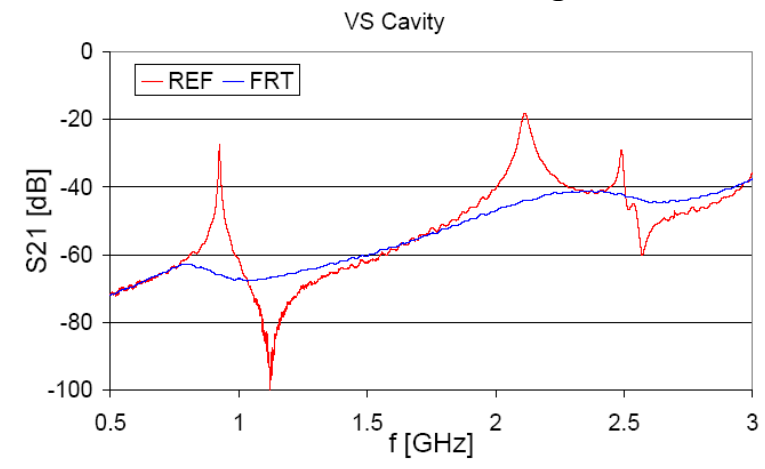

Figure 6: S21 data for reference (red) and ferrite loaded (blue) VS cavity 


\section{TM010 resonance in the MWS eigenvalue solver}

The quality factor of a resonance can be obtained directly from the S21 measurements using the network analyzer 3-dB option or by extraction from the smoothed data curve as done in Fig. 7. The superimposed $\sim 1 \mathrm{GHz}$ oscillations are not noise and believed to be resonances within the ferrite tiles. After some averaging, the TM010 resonance in the VS cavity is taken at $\sim 808 \mathrm{MHz}$ with a $Q \approx 3.4$.

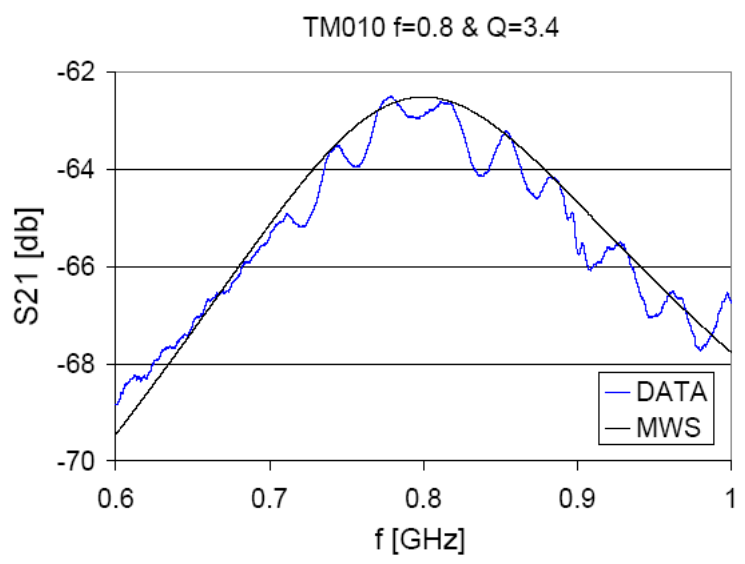

Figure 7. Measured S21 and matched TM010 resonance points

Matching of the resonance can be performed with the JDM eigenmode solver of the MWS simulation program. The frequency and $Q$-values for the tiled model with gap are found for varied $\mu^{\prime}$ and $\tan \delta$ values while keeping a typical $\varepsilon \approx 13$ constant [19]. Selecting sufficiently detailed mesh size at the expense of running time is important. For the VS cavity close to $100 \mathrm{k}$ cells with unchanged settings leads to about an hour for each run. Both frequency shift and $Q$ damping depend primarily on $\mu^{\prime \prime}$, but the use of $\tan \delta$ is more convenient for the simulation program. Using the simulation values for $f$ and $Q$ shown in Fig. 8 , the ferrite parameters were taken as $\mu \approx 4.7(1-j 2.55)$ at the TM010 resonance of $\sim 800 \mathrm{MHz}$.
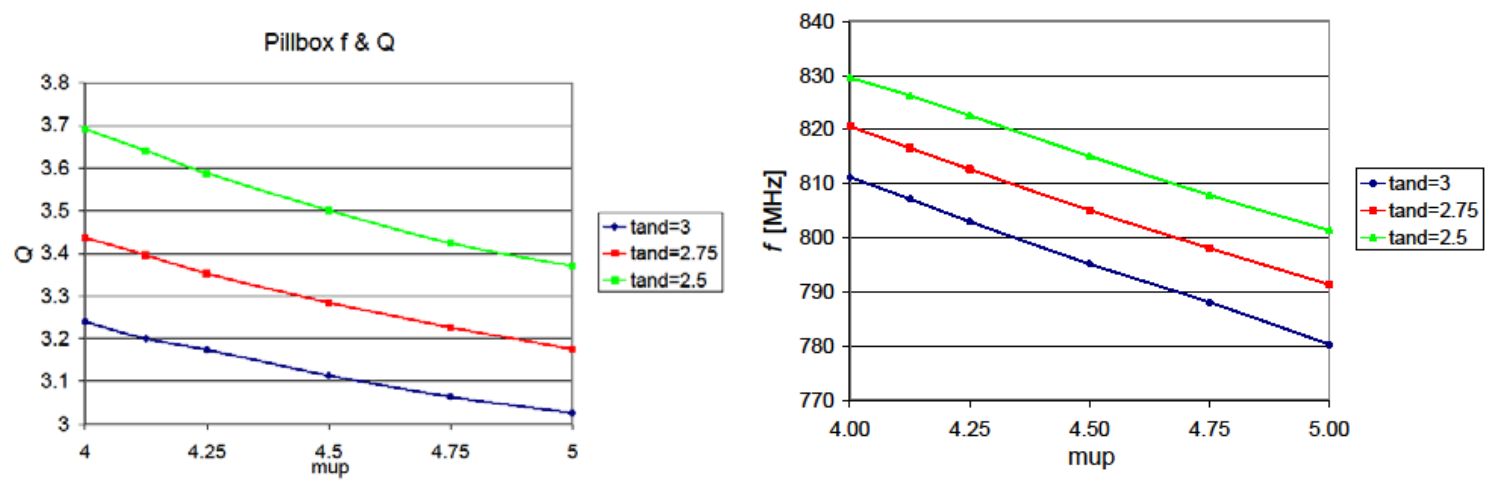

Figure 8: Simulated $Q$ and frequency for different tangent deltas in VS Pillbox cavity 


\section{Simulation with the MWS frequency domain solver}

A more flexible approach of matching the resonance is based on finding the S21 curves with the frequency domain solver over a broader range. For the tiled exact geometry of the VS cavity, a computer run covering the full frequency range from 0.5 to $3 \mathrm{GHz}$ with large mesh numbers $(>100 \mathrm{k})$ for extreme accuracy can last a working day and is not suited to a parameter search. In order to overcome the time limit, it is sufficient to cover the frequency range around the TM010 resonance and only extend the range for a few selected $\mu$ values. The loss contributions from the imaginary permittivity component are small compared to the ferrite losses and all simulations were done with $\varepsilon=13$.

Matching of the measurement with simulation is done here at the resonance peak and the close-by valley as shown in Fig. 9. To allow a comparison, the simulation curves for the reference and the loaded cavity were fitted to the low-frequency slope of the ferrite data. In the figures and the tables, the permeability $\mu^{\prime}(1-j \tan \delta)$ is abbreviated to $\mu^{\prime} J \tan \delta$. The variation of the curves is primarily determined by the choice of $\tan \delta$. A satisfactory match is obtained with $\mu=4.7(1-\mathrm{j} 2.9)$ and a $Q \approx 3.8$, slightly different from the above eigenmode solver result.

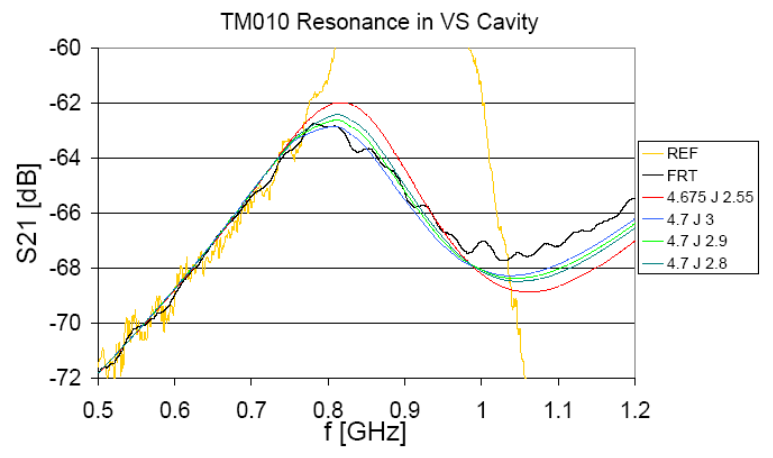

Figure 9. MWS simulation of the TM010 resonance in the VS cavity

\section{First-order Debye model}

The MWS frequency domain solver enters the permeability given by its real part, $\mu^{\prime}$ (mup) and the $\tan \delta$ in a first order Debye model, defined at a specific frequency. It is noted in the MWS simulation that changing the material definition frequency, while maintaining the material parameters at one point changes the $\mu$ and $\tan \delta$ values at other frequencies. The loss factor used in the MWS for different definition frequencies is compared with $\mathrm{MH}$ data in Fig. 10, showing reasonable agreement when $1.5 \mathrm{GHz}$ is chosen. 


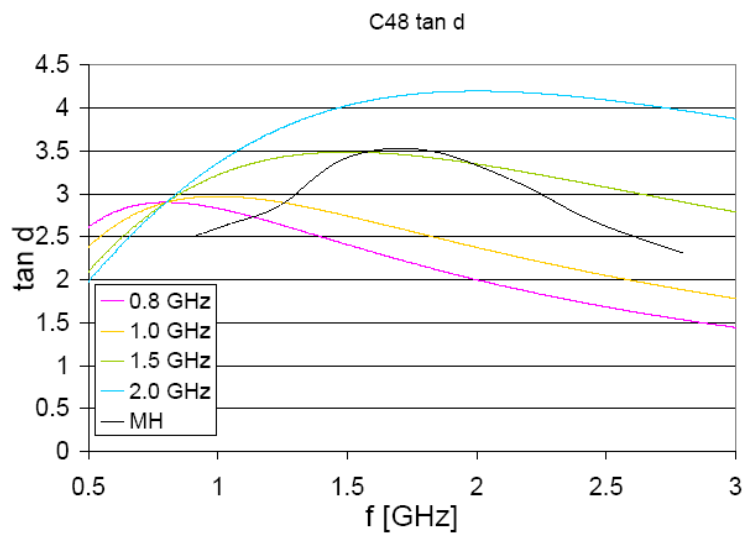

Figure 10: Comparison of $\tan \delta$ in MWS for different definition frequencies with $\mathrm{MH}$ results

The choice of $0.8 \mathrm{GHz}$ for the permeability definition frequency comes naturally at the TM010 resonance. Matching of the S21 measurements with different definition frequencies over the full frequency range was tried leading to the results shown in Fig. 11. The best match, both for the S21 curves in Fig. 11 and the MH permeability in Fig. 12 , is obtained with $\mu=2.2(1-\mathrm{j} 3.5)$ defined at $1.5 \mathrm{GHz}$. The match with the $2 \mathrm{GHz}$ frequency definition, $\mu=1.4(1-\mathrm{j} 4.2)$, gives slightly better agreement for the $\mathrm{S} 21$ comparison but a less plausible loss tangent.

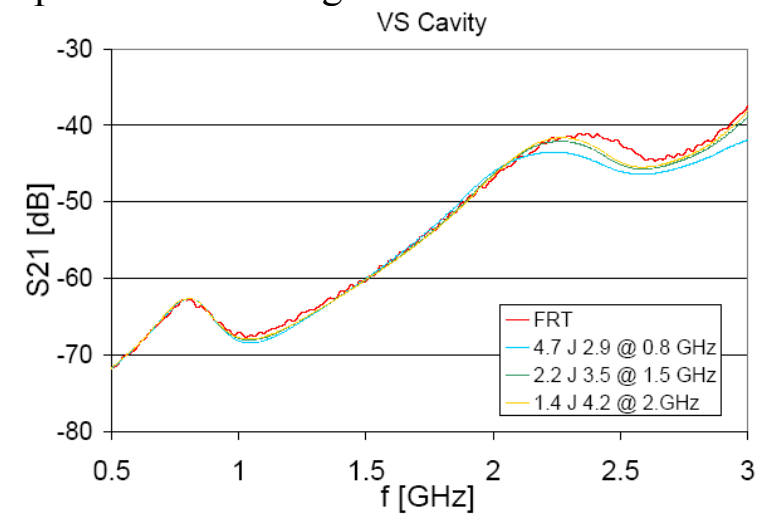

Figure 11. Simulated and measured S21 curves in the VS Cavity.

The permeability, $\mu^{\prime}$ and $\mu^{\prime \prime}$, from this study is compared with $\mathrm{MH}$ data in Fig. 12. The permeability data can be represented by the first order Debye model expression

$$
\mu=1.4+\frac{62.7}{1+j 5.44 f_{G H z}} .
$$

The ferrite permeability data presented here is in reasonable general agreement with the $\mathrm{MH}$ results, although its damping effect is noticeably stronger in the upper frequency range. The small differences may be attributed to the underlying differences in geometries of pellets and tiles. Another error source are the two missing tiles. It is possible that a more general permeability model could provide a better match, but finding it with MWS seems impractical and unnecessary. 


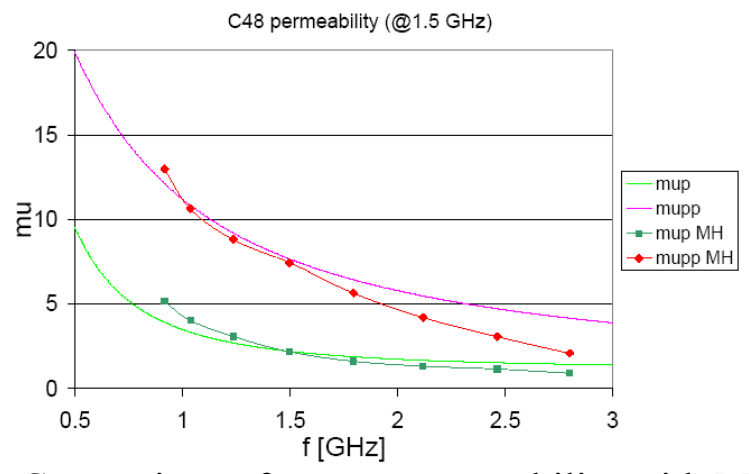

Figure 12. Comparison of present permeability with $\mathrm{MH}$ results.

\section{Geometrical effect of tiled ferrite}

The permeability values can be "ported" to other structures such as the $\mathrm{Cu}$ or the ERL cavities for use in the simulation program, provided that their simulation model has the fully tiled absorber. The effect of the missing tiles and the conductive channels between the tiles is demonstrated in the simulated comparison with a solid ferrite cylinder shown in the Table II. The conductive channels halve the damping properties of the ferrite and the missing tiles significantly reduce the absorber properties. In order to find portable absorber properties a further study involving the "double-cavity", assembled from the prototype absorber with a beam tube section of equal length, became imperative.

Table II. Dependence of frequency and Q-value on ferrite geometry in VS cavity

\begin{tabular}{|c|c|c|c|}
\hline geometry & $\mu @ 0.8 \mathrm{GHz}$ & $f[\mathrm{MHz}]$ & $Q$ \\
\hline Tiled \& gap & $4.7(1-\mathrm{j} 2.6)$ & 803.3 & 3.38 \\
\hline Fully tiled & $4.7(1-\mathrm{j} 2.6)$ & 745.7 & 2.60 \\
\hline Solid & $4.7(1-\mathrm{j} 2.6)$ & 715.6 & 1.28 \\
\hline Solid \& gap & $4.7(1-\mathrm{j} 2.6)$ & 864.9 & 2.66 \\
\hline
\end{tabular}

\section{Ferrite surface impedance}

An alternative way of defining the damping properties of the absorber, although not suited for MWS simulations, is in the form of an equivalent surface resistance, $R_{S}$, which is obtained from the perturbation treatment of the TM010 resonance. The geometry factor of the TM010 mode, assuming losses appearing only on the cylinder, is given as

$$
G=Z_{0} \frac{j_{01}}{2} \approx 453 \Omega
$$

with $j_{01}$ the first root of the Bessel function of zeroth order from which the surface resistance follows by $R_{S}=G / Q$. The surface resistance of the HOM ferrite absorber, estimated as a solid ferrite cylinder, is $R_{S} \approx 133 \Omega$ at $800 \mathrm{MHz}$ compared to $R_{S} \approx 0.06 \Omega$ of stainless steel. An illuminating example of ferrite damping is given by the resulting attenuation $\exp (-\alpha z)$ in the absorber treated as a TM01 waveguide where well above cut-off [23] $\alpha \propto R_{S} / a Z_{0} \approx 3 / \mathrm{m}$. With $a=12 \mathrm{~cm}$ and $Z_{0}=377 \Omega$ one finds $\sim 3 \mathrm{~dB}$ over the $10 \mathrm{~cm}$ length of 2 tiles. 


\section{ABSORBER DOUBLE CAVITY}

The measurements of the fully tiled VS pillbox cavity and their interpretation are presented in the previous section leading to a first-order Debye expression for the complex ferrite parameters valid over the frequency range of interest, here about 0.5 to 3 GHz. In principle, this result is sufficient for use in the MWS simulations to predict the damping of HOMs in the ERL cavity or other cavities. However, it was noted that the geometric absorber details in the VS cavity have a non-negligible impact on the Q-values and a direct measurement of the prototype absorber seemed necessary. Placing shorting plates at the absorber ends leads to overlapping resonances and does not allow a simple interpretation of the network analyzer measurements. To separate the resonances, a "double" (DBL) cavity was formed by adding a beam tube section of equal diameter and length to the absorber and submitted to the standard measurements and MWS simulations.

Running a simulation program for a tiled ferrite structure requires large number of mesh points and in some cases prohibitively long running times. Furthermore simulation programs like Superfish [24] or the SLANS family [25] simulate only rotationally symmetric geometries thus excluding the tiled structure, and the knowledge of $\mu$ is thus of limited value. The primary objective when attaching a ferrite absorber to a cavity is the Q-lowering whereas the change of resonance frequency is typically small and can be ignored. The quest for a simpler, broadly applicable description of the tiled absorber, even at the expense of some loss in accuracy, leads to its replacement by an absorber of cylindrical geometry with properly adjusted material parameters.

The DBL cavity has a sufficiently complex geometry to serve in the comparison of a tiled and solid absorber leading to an equivalent permeability value for the solid ferrite ring. The S21 scattering coefficients for the DBL cavity and a reference cavity are shown in Fig. 13. The first resonance family in the reference cavity, starting at $\sim 920 \mathrm{MHz}$ represents the TM01 modes, and the second, starting at $\sim 2.1 \mathrm{GHz}$, shows the TM02 modes. The first resonance in the ferrite loaded cavity is seen at about $700 \mathrm{MHz}$ but too damped for firm identification as TM010. The second resonance, nominally the TM011 mode, is at $\sim 1 \mathrm{GHz}$ with $Q \approx 35$.

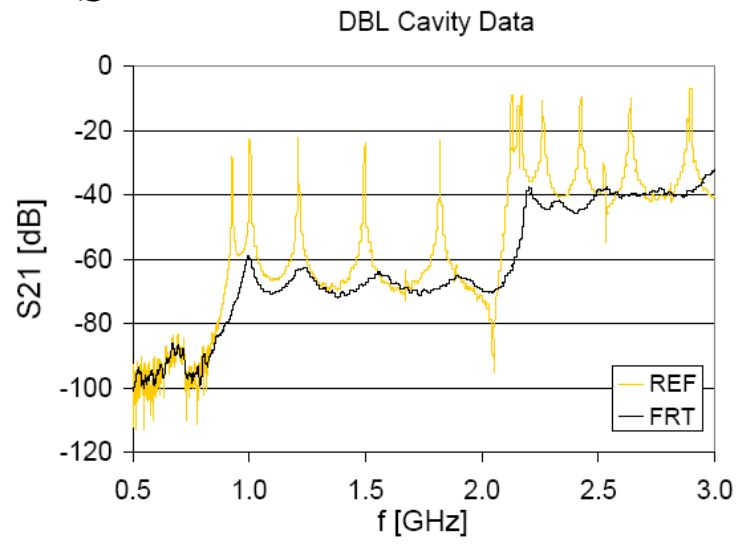

Figure 13. S21 scattering coefficient in the DBL cavity 
The simulation results for the first two modes in the DBL cavity with fully tiled and solid ferrite ring are listed in Table III. In the figures and the tables, the permeability $\mu^{\prime}(1-j \tan \delta)$ is abbreviated to $\mu^{\prime} J \tan \delta$. At the expense of reduced accuracy and based on the TM011 mode only, the equivalent permeability of the solid ferrite absorber can be quoted as $\mu=4(1-\mathrm{j} 2.5)$ defined at $1 \mathrm{GHz}$.

Table III. Comparison of tiled and solid absorber in the MWS simulation

\begin{tabular}{|c|c|c|c|c|c|c|}
\hline & $\mu$ & $@[\mathrm{GHz}]$ & $f[\mathrm{MHz}]$ & $Q$ & $f[\mathrm{MHz}]$ & $Q$ \\
\hline & & & TM010 & & TM011 & \\
\hline tiled & 4.7 J 2.55 & 0.8 & 843 & 3.1 & 974 & 42.7 \\
\hline tiled & 4 J 2.5 & 1 & 869 & 3.6 & 975 & 38.2 \\
\hline solid & 4.7 J 2.55 & 0.8 & 831 & 1.6 & 934 & 40.1 \\
\hline solid & 4 J 2.5 & 1 & 867 & 2.3 & 935 & 34.7 \\
\hline
\end{tabular}

The quasi-suppression of the TM010 mode and the relatively weak damping of the TM011 mode can be explained via the simulated field shapes in Fig. 14, where the absolute values of $E_{z}(0)$ and $H_{\perp}(12.2 \mathrm{~cm})$ are shown. In the TM010 mode the fields are concentrated in the absorber section, extending from 0 to $\sim 11 \mathrm{~cm}$, causing the low $Q$ 's. In the TM011 mode the fields are more evenly distributed and the absorber is here less effective allowing a higher $Q$. The TM01 fields in the DBL cavity are highly distorted and cannot be interpreted as a perturbation. The need for a non-perturbative treatment is here indicated by a peculiarity that increasing the loss tangent decreases the resonance damping.

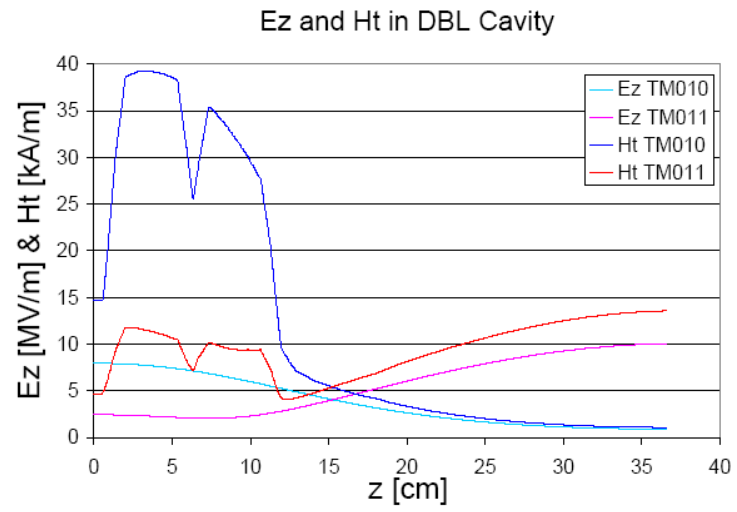

Figure 14. Magnetic and electric field shapes in the Double cavity

The S21 simulation results for the fully tiled and solid ferrite DBL cavity models is compared in Fig. 15 with the measured data over the frequency range from 0.5 to $3 \mathrm{GHz}$. It is noted that the TM010 resonance is not seen in the simulations and the simulation for the TM014 resonance is poor in either model. Overall, one finds sufficient agreement between the fully tiled and solid models and also qualitative agreement with measurements, which allows the conclusion that the tiled C 48 ferrite absorber structure can be replaced in simulations over the full frequency range by solid cylinders with an equivalent permeability of $\mu \approx 4(1-\mathrm{j} 2.5)$ defined at $1 \mathrm{GHz}$. 


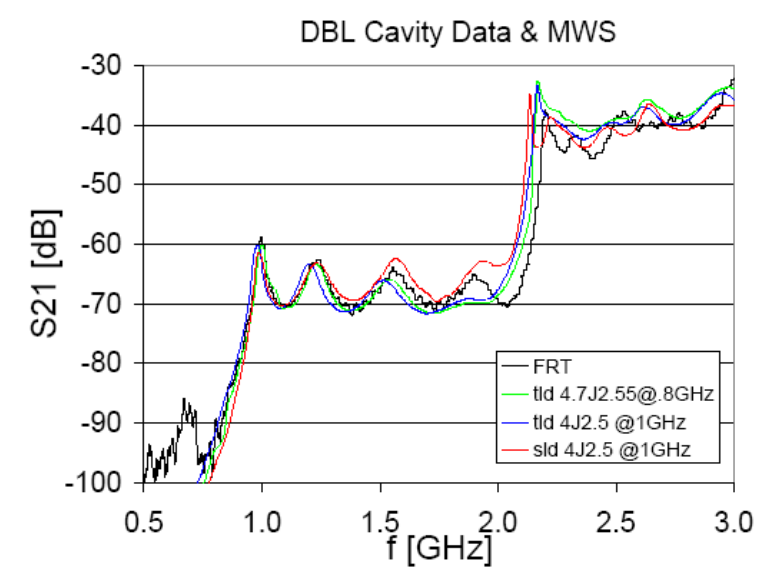

Figure 15. S21 simulation of the DBL box with tiled and solid ferrite

\section{DIPOLE MODES IN THE Cu CAVITY}

The study of the HOM absorber in the previous sections already provides the ferrite parameters required for the simulation of the superconducting ERL cavity and the prediction of its HOM $Q$ and $R / Q$-values. In this section, a confirmation of the ferrite data is obtained from the measurement plus simulation of the simpler but fully representative case of the 5 cell copper cavity.

Minimizing the transverse emittance growth due to the HOMs in the ERL is the primary motivation for the ferrite absorbers. Studying the absorber damping of the dipole modes in the copper prototype is best done by means of the S21 scattering coefficient between two PU probes. Placing the probes in one plane at the cavity side ensures the mode polarization and gives accurate quality factors. The measured S21 signal between the probes in the $\mathrm{Cu}$ cavity assembled with the ferrite absorber or as reference with a beam tube of equal length is shown in Fig. 16. The general character of the hybrid dipole modes is known from the study of the iris-loaded structures used in RF-beam separators [26]. The two lowest pass bands, the TE11 (H11)-like mode from 806 to $896 \mathrm{MHz}$ and the TM11 (E11)-like mode from 958 to $993 \mathrm{MHz}$, are clearly distinguishable. 


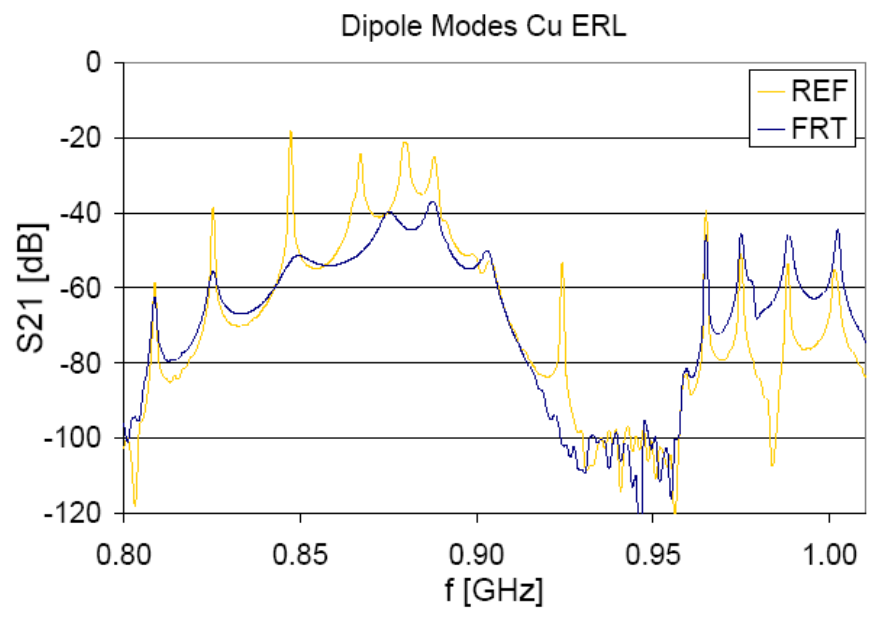

Figure 16. HOM Dipole modes without and with absorber

\section{The bare copper cavity}

In order to establish a solid basis, the bare cavity without beam tube or absorber was simulated in the MWS. The relevant properties from the MWS simulation (indexed MWS) and the measured frequency values (indexed Data) for the bare cavity are listed in Table IV. A full agreement between data and simulation is prevented by differences in cavity geometry and simulation model, and an agreement of better than $\sim 1 \%$ cannot be expected. The measured $Q$ data are all above $\sim 10,000$ but joint losses keep them below the MWS perturbation value for a copper cavity with $\sigma=5.8 \times 10^{7} / \Omega \mathrm{m}$. The prominent field component in each cell is identified as " $\mathrm{H}$ " or " $\mathrm{E}$ ", with capital or lower case letters if strong or weak. It is to be noted that the $\pi$-mode is precluded in the TM11-like pass band. In spite of these limitations, the HOMs in the bare copper cavity are valuable input for mode identification in the copper cavity with the beam tubes and absorber attached.

Table IV. Dipole Pass Bands in the Cu cavity

\begin{tabular}{|c|c|c|c|r|r|c|c|c|c|c|}
\hline $\begin{array}{c}f_{\text {Data }} \\
{[\mathrm{MHz}]}\end{array}$ & $\begin{array}{c}f_{\text {MWS }}[\mathrm{MHz}] \\
{[\mathrm{MHz}}\end{array}$ & $Q_{\text {Data }}$ & $Q_{\text {Cu }}$ & $\begin{array}{c}R / Q_{\text {Mws }} \\
{[\Omega]}\end{array}$ & $\times \pi / 5$ & $\begin{array}{c}\text { Cell } \\
\# 1\end{array}$ & 2 & 3 & 4 & 5 \\
\hline 808.8 & 806.5 & 23,700 & 27,900 & 0.06 & 0 & & $\mathrm{~h}$ & $\mathrm{H}$ & $\mathrm{h}$ & \\
\hline 825.3 & 823.2 & 17,100 & 28,500 & 0.76 & 1 & $\mathrm{~h}$ & $\mathrm{H}$ & $\mathrm{h}$ & $\mathrm{H}$ & $\mathrm{h}$ \\
\hline 847.3 & 846.5 & 9,800 & 28,800 & 4.30 & 2 & $\mathrm{H}$ & $\mathrm{e}$ & $\mathrm{H}$ & $\mathrm{e}$ & $\mathrm{H}$ \\
\hline 867.1 & 870.7 & 3700 & 28,400 & 43.91 & 3 & $\mathrm{~h}$ & $\mathrm{H}$ & $\mathrm{e}$ & $\mathrm{H}$ & $\mathrm{h}$ \\
\hline 879.2 & 889.3 & 2200 & 27,100 & 74.35 & 4 & $\mathrm{E}$ & $\mathrm{e}$ & $\mathrm{h}$ & $\mathrm{e}$ & $\mathrm{E}$ \\
\hline 888.0 & 896.4 & 1440 & 27,300 & 13.76 & 5 & $\mathrm{E}$ & $\mathrm{E}$ & $\mathrm{E}$ & $\mathrm{E}$ & $\mathrm{E}$ \\
\hline- & - & - & - & - & - & - & - & - & - & - \\
\hline 924.3 & 957.5 & 12,100 & 37,100 & 0.01 & 0 & & $\mathrm{e}$ & $\mathrm{E}$ & $\mathrm{e}$ & \\
\hline 965.0 & 962.6 & 25,100 & 37,400 & 0.62 & 1 & $\mathrm{e}$ & $\mathrm{E}$ & $\mathrm{h}$ & $\mathrm{E}$ & $\mathrm{e}$ \\
\hline 974.9 & 969.4 & 7600 & 37,400 & 13.14 & 2 & $\mathrm{E}$ & $\mathrm{h}$ & $\mathrm{e}$ & $\mathrm{h}$ & $\mathrm{E}$ \\
\hline 988.1 & 979.1 & 5200 & 42,000 & 6.5 & 3 & $\mathrm{E}$ & $\mathrm{e}$ & $\mathrm{H}$ & $\mathrm{e}$ & $\mathrm{E}$ \\
\hline 1001.3 & 993.3 & 8200 & 50,400 & 0.1 & 4 & $\mathrm{H}$ & $\mathrm{h}$ & $\mathrm{e}$ & $\mathrm{h}$ & $\mathrm{H}$ \\
\hline
\end{tabular}




\section{The copper cavity with absorber}

The measured frequency and $Q$-values (indexed Data) in the copper cavity are listed in Table $\mathrm{V}$ and are juxtaposed to the MWS simulation results for the empty (indexed $\mathrm{Cu}$ ) and the ferrite tiles added (indexed FRT) cavity. In the search of shorter runs, the simulations were done in the JDM eigenmode solver with the permeability defined as $\mu^{\prime}=4.432$ and constant conductivity of $\omega \mu_{0} \mu^{\prime} \tan \delta=78,300 \Omega / \mathrm{m}$ at $900 \mathrm{MHz}$, replacing the Debye approximation $\mu=4(1-\mathrm{j} 2.5)$ at $1 \mathrm{GHz}$. The solver was first run with $\varepsilon=\mu=1$ in the ferrite to locate all 11 dipole resonances, but finding the changes due to the actual lossy situation had to be done by targeting each resonance separately. The frequencies are closely spaced and mode identification was helped by comparing of the $R / Q$ values even though the small asymmetry of the structure distorts the fields in the cells.

The resonance frequency of all modes is determined by the cavity geometry and the effect due to the ferrite absorber in the MWS is only a few percent. Agreement between data and simulation depends to a large part on the accuracy of the model. It is a plausible assumption that the superconducting niobium losses are too small to change the $Q$-values of the HOMs, determined by the ferrite dominated losses, so that in the absence of measured data the simulations can be substituted. The absorber provides better than an order of magnitude damping of the TE11-like dipole, but less for the TM11-like HOMs. The HOM shunt impedances in the SC ERL cavity will be determined primarily by the absorber and thus are already predictable from the present measurements. It is recalled here that trapped modes have been avoided by the "open" cavity design, although at least one mode, apparently with near zero R/Q, seems to remain undamped by the ferrite.

The operationally important $R / Q$ values of the HOMs can in principle be found from bead-pull measurements, an almost impossible task in the presence of overlapping resonances, so that the $R / Q$ values must be found from simulations. The simulation results for $R / Q$ in the copper cavity are only minimally changed by the ferrite and can be used directly if the convergence in the presence of ferrite becomes too slow. If convergence is not achieved the readily found results from lossless ferrite simulations can be used. 
Table V. Measured and Simulated 5-cell Copper cavity

\begin{tabular}{|l|l|l|l|l|l|r|r|}
\hline $\begin{array}{l}f_{\text {Data }} \\
{[\mathrm{MHz}]}\end{array}$ & $\begin{array}{l}f_{\mathrm{Cu}} \\
{[\mathrm{MHz}]}\end{array}$ & $\begin{array}{l}f_{\mathrm{FRT}} \\
{[\mathrm{MHz}]}\end{array}$ & $Q_{\mathrm{Cu}}$ & $Q_{\text {Data }}$ & $Q_{\mathrm{FRT}}$ & $\begin{array}{l}R / Q_{\mathrm{Cu}} \\
{[\Omega]}\end{array}$ & $\begin{array}{l}R / Q_{\mathrm{FRT}} \\
{[\Omega]}\end{array}$ \\
\hline 808.8 & 805.7 & 808.4 & 26,700 & 1780 & 416 & 1.0 & 1.1 \\
\hline 825.3 & 830.0 & 833.9 & 27,600 & 415 & 151 & 3.0 & 3.4 \\
\hline 848.9 & 852.6 & 847.7 & 28,800 & 120 & 17 & 27.4 & 0.1 \\
\hline 875.7 & 867.2 & 861.8 & 29,100 & 162 & 174 & 59.6 & 33.9 \\
\hline 887.0 & 877.3 & 879.0 & 27,200 & 332 & 1599 & 38.2 & 38.4 \\
\hline 903.1 & 914.0 & 903.3 & 37,700 & 290 & 416 & 13.9 & 13.8 \\
\hline 959.5 & 948.8 & 950.0 & 37,200 & 22,300 & 36,200 & 0.0 & 0.0 \\
\hline 965.0 & 953.3 & 954.7 & 38,500 & 6670 & 7189 & 1.4 & 1.2 \\
\hline 974.9 & 960.3 & 962.1 & 41,200 & 2000 & 2766 & 10.1 & 8.6 \\
\hline 988.1 & 971.0 & 973.1 & 46,600 & 1064 & 1398 & 3.4 & 3.4 \\
\hline 1000.2 & 984.0 & 986.1 & 55,000 & 1512 & 1922 & 1.0 & 1.1 \\
\hline
\end{tabular}

\section{Simulations with tiled and solid ferrite absorber}

Table VI. Simulation of Cu Cavity with Tiled and Solid Absorber

\begin{tabular}{|l|l|l|l|r|r|c|c|}
\hline $\begin{array}{c}f_{\text {tld }} \\
{[\mathrm{MHz}]}\end{array}$ & $\begin{array}{c}f_{\text {sld }} \\
{[\mathrm{MHz}]}\end{array}$ & \multicolumn{1}{|c|}{$Q_{\text {tld }}$} & \multicolumn{1}{|c|}{$Q_{\text {sld }}$} & $\begin{array}{c}R / Q_{\text {tld }} \\
{[\Omega]}\end{array}$ & $\begin{array}{c}R / Q_{\text {sld }} \\
{[\Omega]}\end{array}$ & $R_{\text {tld }}[\Omega]$ & $R_{\text {sld }}[\Omega]$ \\
\hline & 802.3 & & 1969 & & 0.1 & & \\
\hline 808.4 & 820.3 & 416 & 591 & 1.1 & 0.9 & 458 & 532 \\
\hline 833.9 & 845.2 & 151 & 234 & 3.4 & 4.6 & 513 & 1076 \\
\hline 847.7 & 872.4 & 17 & 21 & 0.1 & 0.1 & 2 & 2 \\
\hline 861.8 & 875.6 & 174 & 128 & 33.9 & 25.0 & 5899 & 3200 \\
\hline 879.0 & 891.7 & 1599 & 1077 & 38.4 & 31.1 & 61,400 & 33,495 \\
\hline 903.3 & 916.4 & 41.6 & 33 & 13.8 & 10.7 & 574 & 353 \\
\hline 950.0 & 960.4 & 36,200 & 66,330 & 0.0 & 0.0 & 362 & 398 \\
\hline 954.7 & 965.1 & 7189 & 11,070 & 1.2 & 1.5 & 8627 & 16,605 \\
\hline 962.1 & 971.8 & 2766 & 4402 & 8.6 & 10.3 & 23,788 & 45,341 \\
\hline 973.1 & 981.3 & 1398 & 1978 & 3.4 & 3.4 & 4753 & 6725 \\
\hline 986.1 & 993.1 & 1922 & 2701 & 1.1 & 1.2 & 2114 & 3241 \\
\hline
\end{tabular}

Simulations of the $\mathrm{Cu}$ cavity also provided the dipole $Q$ - and $R / Q$ values for the models with a fully tiled (indexed tld) and solid (indexed sld) ferrite absorber. The models are based on an identical cell structure, but the treatment for the tiles and the ferrite ring differs which may be the reason for some of the differences. The MWS simulation results for the dipole HOMs obtained with equal ferrite parameters are listed in Table VI. Mode identification in the case of narrow resonances is aided by $R / Q$ matching. The results confirm that the tiled structure can be substituted with a solid ring without significant loss of accuracy. It must be recorded without further discussion that several additional resonances located in the beam tube directly at the ferrite were found which have $Q$ values of a few tens and very low shunt impedance. 


\section{Shorted and open absorber end}

The copper cavity was tested with the absorber end shorted (indexed shrt) and open (indexed opn), reflecting a step or in the latter case the presence of a taper. Table VII presents the measured frequency and Q-values (indexed Data) as well as the MWS simulations for the shorted and "waveguide" terminated model. Finding eigenvalues in the open model proved to be difficult and not all were found. The $\sim 960 \mathrm{MHz}$ resonance in all models has the character of a trapped mode, albeit with a very small shunt impedance. Overall, an increased damping from using a taper is either marginal or nonexistent, and the benefit of a shorter cavity length in the ERL justified the adopted step transition at the absorber end.

Table VII. Measured and simulated results for the shorted and open $\mathrm{Cu}$ Cavity

\begin{tabular}{|l|l|l|l|l|l|r|r|}
\hline $\begin{array}{l}f_{\text {Data }}^{\text {shrt }} \\
{[\mathrm{MHz}]}\end{array}$ & $\begin{array}{l}f_{\text {Data }}^{\text {opn }} \\
{[\mathrm{MHz}]}\end{array}$ & $Q_{\text {Data }}^{\text {shrt }}$ & $Q_{\text {Data }}^{\text {opn }}$ & $Q_{\mathrm{FRT}}^{\text {shrt }}$ & $Q_{\mathrm{FRT}}^{\text {opn }}$ & \multicolumn{1}{|l|}{$\begin{array}{l}R / Q^{\text {shrt }} \\
{[\Omega]}\end{array}$} & \multicolumn{2}{|c|}{$\left[/ Q^{\text {opn }}\right.$} \\
& & & & 1969 & 1152 & 0.1 & 0.1 \\
\hline 808.4 & 808.8 & 1780 & 670 & 591 & 263 & 0.9 & 0.6 \\
\hline 833.9 & 826.4 & 415 & 490 & 234 & 203 & 4.6 & 6.5 \\
\hline 847.7 & 849.5 & 120 & 300 & 21 & & 0.1 & \\
\hline 861.8 & 873.7 & 162 & 211 & 128 & 326 & 25.0 & 44.6 \\
\hline 879.0 & 886.9 & 332 & 275 & 1077 & 1849 & 31.1 & 38.8 \\
\hline 903.3 & 901.2 & 290 & 730 & 33 & 73 & 10.7 & 15.1 \\
\hline 950.0 & 959.5 & 22,300 & 12,160 & 66,330 & 72,224 & 0.0 & 0.0 \\
\hline 954.7 & 965.0 & 6670 & 2815 & 11,070 & 10,171 & 1.5 & 1.3 \\
\hline 962.1 & 974.9 & 2000 & 890 & 4402 & 3694 & 10.3 & 10.9 \\
\hline 973.1 & 988.1 & 1064 & 586 & 1978 & 1263 & 3.4 & 3.8 \\
\hline 986.1 & 1001.3 & 1512 & 1033 & 2701 & 1271 & 1.2 & 1.2 \\
\hline
\end{tabular}

\section{HOMs IN THE RHIC ERL CAVITY}

The recently gained availability of the ERL cavity shown in Fig. 1, even prior to assembly in its cryostat, provided an excellent opportunity to compare measured HOM data with predictions based on the ferrite properties derived from the copper prototype. The niobium cavity differs from the $\mathrm{Cu}$ model primarily by the presence of two ferrite HOM absorbers and their significantly increased distances from the cavity ends.

The niobium cavity is equipped with 3 button probes (with Ceramaseal SMA feedthrough) at both room temperature cavity ends which are intended for beam orbit positioning and allow scattering coefficient measurements of HOMs. The buttons provide HOM signals without regard to their polarity and yield HOM data but are not reached by the fundamental mode. The fundamental mode could only be measured between the fundamental power coupler (FPC) and the pickup (PU) probe in the cavity planned for the RF control system. The room temperature fundamental frequency in the ERL cavity is found to be $702.43 \mathrm{MHz}$ with a $Q$ of 11,000. Cool down will increase the frequency by $1.035 \mathrm{MHz}$ bringing it to $703.465 \mathrm{MHz}$ which is within the slow-tuner range for the Coherent Electron Cooling operation at $703.5369 \mathrm{MHz}$. 
The HOMs are studied via S21 measurements between the access points in several configurations, the one between FPC and PU is shown in Fig. 17. The frequency range covers the lowest dipole and monopole modes, from 0.8 to $1 \mathrm{GHz}$ and from 1.2 to 1.6 $\mathrm{GHz}$ respectively. Although the resonances are all strongly damped, $Q$-values for some modes have been measured but identification depends on eigenvalue results with lossy ferrite which could not be found above $\sim 1 \mathrm{GHz}$ with the MWS simulation program.

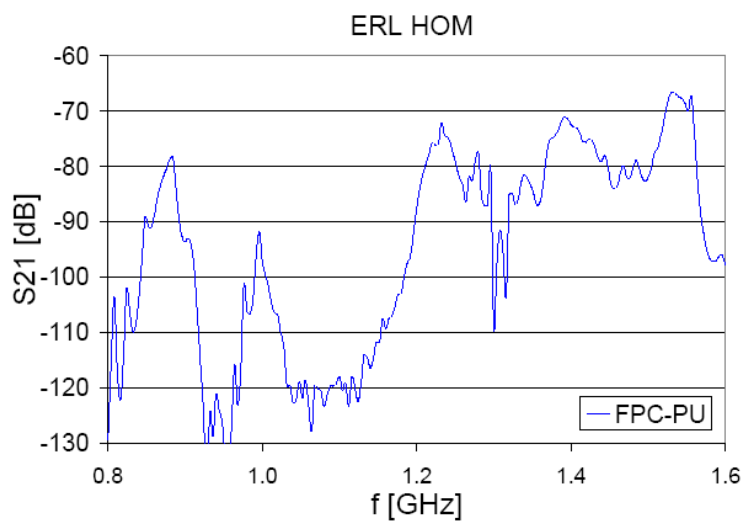

Figure 17: S21 from FPC to PU

\section{Dipole HOMs}

Table VIII. Dipole resonances in the RHIC ERL cavity

\begin{tabular}{|l|l|r|l|l|r|l|l|r|r|}
\hline $\begin{array}{l}f_{\mathrm{cu}} \\
{[\mathrm{MHz}]}\end{array}$ & $Q_{\mathrm{cu}}$ & $\begin{array}{l}R / Q_{\mathrm{cu}} \\
{[\Omega]}\end{array}$ & $\begin{array}{l}f_{\mathrm{FRT}} \\
{[\mathrm{MHz}]}\end{array}$ & $Q_{\mathrm{FRT}}$ & $\begin{array}{l}R / Q_{\mathrm{FRT}} \\
{[\Omega]}\end{array}$ & $\begin{array}{l}f_{\text {Data }} \\
{[\Omega]}\end{array}$ & $Q_{\text {Data }}$ & $\begin{array}{l}R_{\mathrm{cu}} \\
{[\mathrm{k} \Omega]}\end{array}$ & $\begin{array}{l}R_{\mathrm{FRT}} \\
{[\mathrm{k} \Omega]}\end{array}$ \\
\hline 805.1 & 29,700 & 0.0 & 803.4 & 588 & 0.1 & & & 1.1 & 0.08 \\
\hline 810.2 & 34,000 & 0.4 & 808.4 & 150 & 1.1 & 807.8 & 900 & 12.7 & 0.17 \\
\hline 817.9 & 34,500 & 1.2 & 816.1 & 136 & 0.7 & & & 40.6 & 0.10 \\
\hline 829.2 & 32,000 & 0.3 & 827.0 & 265 & 0.2 & 825.18 & 370 & 10.2 & 0.06 \\
\hline 851.6 & 31,000 & 13.7 & 849.5 & 493 & 11.8 & 848.99 & 130 & 426.2 & 5.84 \\
\hline 874.6 & 31,100 & 55.1 & 872.6 & 379 & 49.3 & & & 1712.9 & 18.70 \\
\hline 890.2 & 32,800 & 43.0 & 888.1 & 163 & 42.0 & & & 1411.0 & 6.85 \\
\hline 897.1 & 31,900 & 21.2 & 895.0 & 201 & 22.8 & & & 675.9 & 4.58 \\
\hline- & - & - & - & - & - & - & - & & - \\
\hline 929.0 & 42,800 & 10.1 & 926.2 & 104 & 6.7 & & & 433.3 & 0.69 \\
\hline 943.8 & 46,000 & 5.8 & 940.7 & 79 & 8.6 & & & 266.7 & 0.68 \\
\hline 957.6 & 38,500 & 0.1 & 956.1 & 40,370 & 0.0 & 958.34 & 9,500 & 2.0 & 1.57 \\
\hline 963.7 & 40,700 & 2.6 & 962.2 & 6410 & 2.6 & 964.76 & 3,350 & 107.5 & 16.98 \\
\hline 975.4 & 45,800 & 7.9 & 973.7 & 2132 & 8.1 & 977.15 & 830 & 361.5 & 17.21 \\
\hline 991.8 & 54,200 & 2.4 & 989.8 & 1644 & 2.4 & 995.46 & 205 & 130.5 & 3.92 \\
\hline
\end{tabular}

Several dipole modes at frequencies below $1 \mathrm{GHz}$ were measured and could be identified by comparison with simulation results. For this purpose, the bare ERL model was expanded by adding the beam tubes and both ferrite absorbers. Two simulation runs were made for which the ferrite was first treated as vacuum and then given by $\mu=4$ (1- 
j2.5) defined at $1 \mathrm{GHz}$. The effect of the ferrite on the resonance frequencies is small, less than $\sim 3 \%$ in frequency, but slightly higher in the $R / Q$ values. The damping of the $Q$-values is almost 2 orders of magnitude for the TE11-like modes but can be significantly lower for some of the TM11-like modes. The simulation results, including the projected shunt impedance $R_{F R T}$ values are listed in Table VIII. Measured dipole modes were identified by referring to the simulation results and are also given in this table. The reasonable agreement between simulated values and measured data justifies the use of the simulated shunt impedance for all dipole resonances.

\section{Monopole HOMs}

Table IX :ERL Monopole modes in the GHz region

\begin{tabular}{|c|c|r|r|}
\hline BARE & & STRING & \\
\hline$f \mathrm{GHz}]$ & $R / Q[\Omega]$ & $f[\mathrm{GHz}]$ & \multicolumn{2}{|c|}{$R / Q[\Omega]$} \\
\hline & & 1.0471 & 18.05 \\
\hline & & 1.1316 & 24.09 \\
\hline & & 1.1530 & 20.36 \\
\hline & & 1.2349 & 13.09 \\
\hline 1.265 & 97.68 & 1.2658 & 8.22 \\
\hline & & 1.2986 & 0.30 \\
\hline & & 1.3077 & 1.29 \\
\hline 1.3106 & 0.49 & 1.3210 & 0.54 \\
\hline & & 1.3368 & 4.59 \\
\hline 1.3459 & 2.73 & 1.3525 & 0.85 \\
\hline & & 1.3711 & 3.07 \\
\hline & & 1.3918 & 8.14 \\
\hline 1.3961 & 2.98 & 1.4123 & 1.94 \\
\hline & & 1.4410 & 0.34 \\
\hline & & 1.4744 & 7.94 \\
\hline 1.4649 & 6.18 & 1.4973 & 10.09 \\
\hline & & 1.5209 & 16.02 \\
\hline & & 1.5209 & 16.02 \\
\hline 1.5316 & 3.36 & 1.5438 & 31.86 \\
\hline
\end{tabular}

In the $\mathrm{GHz}$ region, monopole and higher-order pole pass bands can overlap. HOM identification, by bead-pull measurement in the ERL is impractical, and by simulation seems to exceed the capability of the MWS. Furthermore, the search for other programs is beyond the scope of this paper. Nevertheless a monopole pass band with six resonances, one located in the beam tube, has been found from simulation between $\sim 1.2$ and $1.5 \mathrm{GHz}$ for the bare, without ferrite, ERL cavity. The number of monopole modes in this region increases to 18 for the simulation of the ERL string without ferrite. Here, about 22 quadrupole modes appear in addition. The simulation frequencies and monopole $R / Q$ values are listed in Table IX as reference for future studies with an ebeam. All $Q$ values from simulation and the few measured in the ERL string are shown in Fig. 18. 


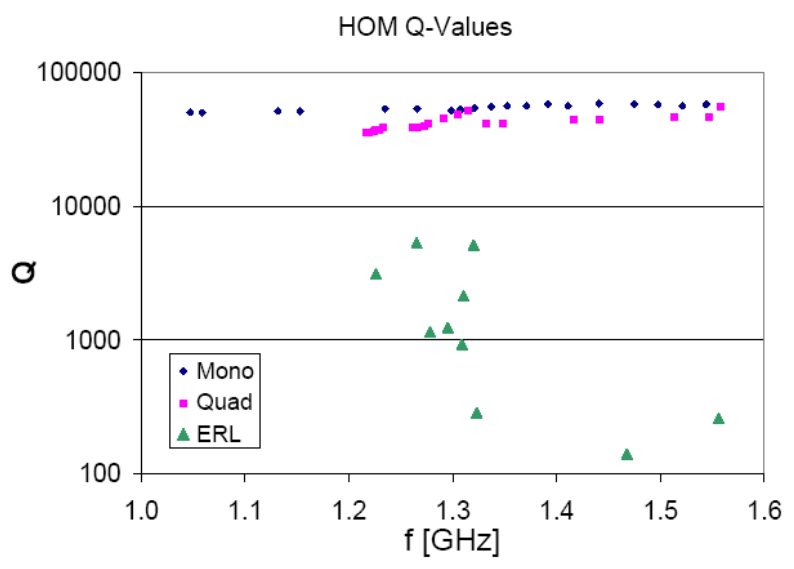

Figure 18. Measured and simulation $Q$-values of ERL HOMs (Blue circles and pink squares represent simulations and green triangles represent measurements.)

In conclusion, even though only some of the dipole modes have been identified, the totality of the results confirms the effective damping of HOMs in the ERL by the ferrite absorber

\section{Acknowledgements}

The authors would like to thank Drs. Ilan Ben-Zvi and Vladimir Litvinenko for helpful comments. The support provided by the Collider-Accelerator technical staffing setting up the cavities was greatly appreciated.

This work was supported by Brookhaven Science Associates, LLC under Contract No. DE-AC02-98CH10886 with the U.S. Department of Energy. The publisher by accepting the manuscript for publication acknowledges that the United States Government retains a non-exclusive, paid-up, irrevocable, world-wide license to publish or reproduce the published form of this manuscript, or allow others to do so, for United States Government purposes. 


\section{References}

1. I. Ben-Zvi, Proc. $13^{\text {th }}$ International Workshop on RF Superconductivity 2007, Beijing, China, paper FR101

2. V. Litvinenko et al., Proc. EPAC 2008, Genoa, Italy, p.193

3. D. Moffat, et al., Proc PAC 1991, San Francisco, CA, p. 664

4. S. Belomestnykh, et al., Proc PAC 1995, Dallas TX, p. 3394

5. T. Tajima, et al., Proc PAC 1999, New York, NY, p. 440

6. R. Calaga, Linear Beam Dynamics and Ampere Class Superconducting RF Cavities at RHIC, (Dissertation, SUNY Stony Brook, 2006)

7. Advanced Energy Systems, Medford, New York, 11763

8. ACCEL Instruments GmbH, 51429 Bergisch-Gladbach, Germany

9. V. Shemelin, Transverse Hybrid Mode in a Ferrite-Lined Pillbox Cavity. Comparison of Theory and Codes: CLANS2, MWS et al., Report ERL 08-1 (Cornell University, 2008)

10. CST-Computer Simulation Technology, Darmstadt, Germany

11. H. Hahn, et al., Physica C, 239 (2006)

12. H. Hahn, et al., ref. 2, p. 3705

13. H. Hahn, et al., Ferrite Lined Pill box, Report C-AD/AP/269 (BNL, 2007)

14. R. Calaga, et al., Report C-AD/AP/111 (Collider-Accelerator Department, BNL, 2003)

15. E. Chojnacki and W. J. Alton, ref. 5, p. 845

16. I. E. Campisi and L.R. Doolittle, Eds., Proceedings of the First Workshop on Microwave-Absorbing Materials for Accelerators, CEBAF, Newpoer News, VA, 1993, and I.E. Campisi, Proc. PAC 1993, Washington, DC, p.1115

17. W. H. Hartung, Proc. PAC 1993, p. 3450; The Interaction Between a Beam and a Layer of Microwave-Absorbing Material, Thesis (Cornell University, 1996)

18. T. Tajima, et al., ref. 4, p. 1620

19. Countis Laboratories, 12295 Charles Drive, Grass Valley CA 95945

20. J. Mouris and R. M. Hutcheon, Measurements of the complex microwave permeability of un-biased Ferrite C-48 and Ferrite-50, from room temperature to $200{ }^{\circ} \mathrm{C}$ at frequencies between $915 \mathrm{MHz}$ and $2800 \mathrm{MHz}$., Report MPN-41-00

(Canadian Light Source / Microwave Properties North, December 2000)

21. V. Shemelin et al., NIM in Physics Research, A557, p. 268 (2006)

22. H. Padamsee, J. Knobloch, and T. Hays, RF Superconductivity for Accelerators, (John Wiley \& Sons, Inc, New York 1998), p.133

23. N. Marcuvitz, ed., Waveguide Handbook, (McGraw Hill Book Co, New York, 1951), p. 67

24. Poisson Superfish, http://laacg1.lanl.gov/laacg/services/download_sf.phtml

25. D. G. Mayakishev and V. P. Yakolev, ref. 5, p. 2775

26. H. Hahn, Rev. Sci. Instruments, 34, 1094 (1963) 


\section{APPENDIX: Skin effect analysis}

The ferrites are flat tiles whose dimensions are very small compared with the beam tube radius so that the electromagnetic (e. m.) fields in the ferrite can be analyzed in a Cartesian coordinate system in full analogy to the "classical" skin effect treatment. The fields in the material are locally driven by a magnetic field $H_{y}(z=0)=1$ which is due to an incoming TEM wave perpendicular to the $x-y$ surface and is position independent over the inner surface. The time-harmonic electric field in the ferrite material is derived from the solution of the wave equation

$$
\nabla^{2} H+\mu \varepsilon_{S} k^{2} H=0
$$

with the material parameters $\mu=\mu^{\prime}-j \mu^{\prime \prime}, \varepsilon_{S}=\varepsilon^{\prime}-j\left(\varepsilon^{\prime \prime}+S\right), S=\sigma /\left(\omega \varepsilon_{0}\right)$, and the free space wave number $k=\omega / c$. Taking into account the boundary condition $E_{x}(z=\tau)=0$ of a perfect conductor at the outer ferrite wall, the field expressions are found as follows

$$
\begin{aligned}
& E_{x}=-j \frac{\kappa}{\varepsilon_{S} k} \sinh \kappa(\tau-z) \\
& Z_{0} H_{y}=\cosh \kappa(\tau-z)
\end{aligned}
$$

with the ferrite wave number $\kappa^{2}=-\mu \varepsilon_{S} k^{2}$. The field solution yields the expression for the complex surface impedance in the $\mathrm{x}-\mathrm{y}$ plane

$$
\mathfrak{R}=\frac{E_{x}}{H_{y}}=Z_{0} \sqrt{\frac{\mu}{\varepsilon_{S}}} \tanh \kappa \tau .
$$

Although generally valid, both for the high-conductivity metals as well as the ferrite parameters considered here, it is instructive to consider them separately.

\section{The skin effect in metal}

The field penetration depth in metals is usually much smaller than the wall thickness of metal so that $\tanh \kappa \tau \approx 1$ and the conductivity in metals dominates permittivity, $S \gg \varepsilon^{\prime}, \varepsilon^{\prime \prime}$, and $\mu^{\prime} \gg \mu^{\prime \prime}$, leading to

$$
\Re_{\infty} \approx Z_{0} \sqrt{j \mu^{\prime} / S}=(1+j) \sqrt{\mu^{\prime} \mu_{0} \omega / 2 \sigma},
$$

and the skin depth

$$
\delta=\frac{1}{\operatorname{Re} \kappa} \approx \frac{1}{k} \sqrt{\frac{2}{\mu^{\prime} S}}=\sqrt{\frac{2}{\mu^{\prime} \mu_{0} \omega \sigma}}
$$

in full agreement with the well-known "classical" results.

\section{Skin effect in flat ferrite tiles}

The material parameters of ferrite differ qualitatively from a metal, notably due to $\sigma \sim 0$, and require a separate discussion. However, in the frequency range of interest, the material is in good approximation determined by generic ferrite properties with only $\mu^{\prime}, \mu^{\prime \prime}, \varepsilon^{\prime}$. Measured permeability values provide adequate numerical results for the flat ferrite approximation. The complex surface impedance of a ferrite tile with finite thickness, $\tau$, and attached to a perfect conductor is given by 


$$
\mathfrak{R}=Z_{0} \sqrt{\frac{\mu^{\prime}-j \mu^{\prime \prime}}{\varepsilon^{\prime}}} \tanh \left(k \tau \sqrt{-\left(\mu^{\prime}-j \mu^{\prime \prime}\right) \varepsilon^{\prime}}\right)
$$

and the "nominal" skin depth by

$$
\delta=\frac{1}{\operatorname{Re} \kappa}=\frac{1}{k \operatorname{Re} \sqrt{\left(j \mu^{\prime \prime}-\mu^{\prime}\right) \varepsilon^{\prime}}}
$$

In contrast to a metal, the magnetic field fully penetrates the ferrite and changes the surface impedance by the tanh $\kappa \tau$ factor. Taking the wall thickness as $\tau=3 \mathrm{~mm}$ and the measured ferrite permeability, $\mu=1.4+62.7 /\left(1+j 5.44 f_{G H z}\right)$, one finds the correction factor shown in Fig. 19. It is seen that a finite wall thickness has a non-negligible impact in the frequency region of interest.

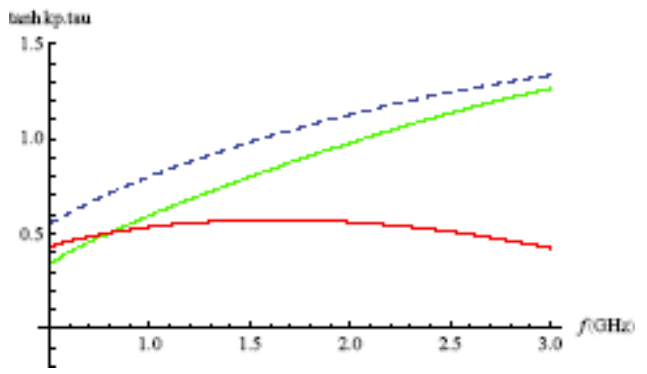

Figure 19. The correction factor $\tanh \kappa \tau$ versus frequency, with real (green), imaginary (red), and absolute (dashed) value.

In the lower frequency region of interest relevant to the present study, one has $\tanh \kappa \tau \approx \kappa \tau$, leading to the approximate expression for the surface impedance

$$
\mathfrak{R} \approx j Z_{0} \mu k \tau
$$

in which the dielectric properties disappear. In the upper frequency region, one approaches the case of $\tanh \kappa \tau \sim 1$. The Surface resistance, $R_{s}=\operatorname{Re} \mathfrak{R}$, of a $3 \mathrm{~mm}$ thick ferrite is computed with Mathematica for the measured permeability and shown in Fig. 21 for the three cases of the full expression equ. A6 (green), the approximation $\tanh \kappa \tau \approx \kappa \tau$ (red), and the limit at $\tanh \kappa \tau \sim 1$ (green, dashed). The three curves are not close together and the use of approximations can be misleading. It is noted that the calculated surface resistance is about a factor 2 larger than the measured one from the TM010 resonance, which is attributed to the tiled structure in the VS pillbox.

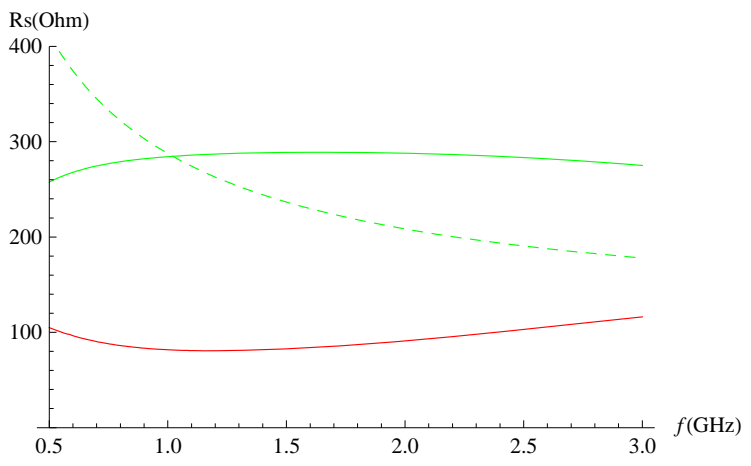

Figure 20. Surface resistance at the $3 \mathrm{~mm}$ thick ferrite tile the full expression (green), the low $-\mathrm{f}$ (red) and high-f (green, dashed) approximation. 


\section{Skin effect in circular cylindrical geometry}

For the purpose of a theoretical analysis of wave propagation in the HOM ferrite absorber, the actual tiled shape is replaced by a circular ferrite cylinder in a lossless waveguide. The inner radius of the ferrite is $a$ and the outer radius $b$ is touching the circular waveguide. The time-harmonic fields in the ferrite-free inner region, with the time factor $e^{j \omega t}$ omitted, are given by

$$
\begin{gathered}
E_{z i}=\frac{k^{2}-k_{z}^{2}}{k_{r i} k} J_{0}\left(k_{r i} r\right) e^{-j k_{z} z} \\
E_{r i}=j \frac{k_{z}}{k} J_{1}\left(k_{r i} r\right) e^{-j k_{z} z} \\
Z_{0} H_{\Theta i}=j J_{1}\left(k_{r i} r\right) e^{-j k_{z} z}
\end{gathered}
$$

where the wave numbers are obviously $k=\omega / c, k_{z}$, and $k_{r i}$. SI units are used with $c=1 / \sqrt{\varepsilon_{0} \mu_{0}}$ and $Z_{0}=\sqrt{\mu_{0} / \varepsilon_{0}}$. The field components in the ferrite have the same propagation constant, $e^{-j k_{z} z}$, and must satisfy the boundary condition $E_{z}(b)=0$, be continuous in $E_{z}$ and $H_{\Theta}$ at $r=a$, and can be written as

$$
\begin{aligned}
& E_{z o}=\frac{\varepsilon_{S} \mu k^{2}-k_{z}^{2}}{\varepsilon_{S} k_{r o} k} \frac{J_{1}\left(k_{r i} a\right)}{F_{1}\left(k_{r o} a\right)} F_{0}\left(k_{r o} r\right) e^{-j k_{z} z} \\
& E_{r o}=j \frac{k_{z}}{\varepsilon_{S} k} \frac{J_{1}\left(k_{r i} a\right)}{F_{1}\left(k_{r o} a\right)} F_{1}\left(k_{r o} r\right) e^{-j k_{z} z} \\
& Z_{0} H_{\Theta o}=j \frac{J_{1}\left(k_{r i} a\right)}{F_{1}\left(k_{r o} a\right)} F_{1}\left(k_{r o} r\right) e^{-j k_{z} z}
\end{aligned}
$$

with the combinations of Bessel-functions

$$
\begin{aligned}
& F_{0}\left(k_{r o} r\right)=J_{0}\left(k_{r o} r\right) Y_{0}\left(k_{r o} b\right)-J_{0}\left(k_{r o} b\right) Y_{0}\left(k_{r o} r\right) \\
& F_{1}\left(k_{r o} r\right)=J_{1}\left(k_{r o} r\right) Y_{0}\left(k_{r o} b\right)-J_{0}\left(k_{r o} b\right) Y_{1}\left(k_{r o} r\right)
\end{aligned}
$$

and the relative material parameters $\mu=\mu^{\prime}-j \mu^{\prime \prime}$ and $\varepsilon_{S} \approx \varepsilon^{\prime}$.

Finding a solution involves satisfying three simultaneous conditions. Vanishing divergence, $\nabla E=0$, provides two conditions on the wave numbers,

$$
k^{2}-k_{r i}^{2}-k_{z}^{2}=0 \text { and } \varepsilon_{S} \mu k^{2}-k_{r o}^{2}-k_{z}^{2}=0
$$

and continuity of $E_{z}$ at $r=a$ yields the necessary third ( $H_{\Theta}$ at $r=a$ is built-in),

$$
\frac{k^{2}-k_{z}^{2}}{k_{r i} k} J_{0}\left(k_{r i} a\right)-\frac{\varepsilon_{S} \mu k^{2}-k_{z}^{2}}{\varepsilon_{S} k_{r o} k} \frac{J_{1}\left(k_{r i} a\right)}{F_{1}\left(k_{r o} a\right)} F_{0}\left(k_{r o} a\right)=0
$$

After making an obvious simplification the solutions follows from the two necessary simultaneous equations in $k_{r i}$ and $k_{r o}$,

$$
\begin{aligned}
& \varepsilon_{S} k_{r i} J_{0}\left(k_{r i} a\right) F_{1}\left(k_{r o} a\right)-k_{r o} J_{1}\left(k_{r i} a\right) F_{0}\left(k_{r o} a\right)=0 \\
& \left(\varepsilon_{S} \mu-1\right) k^{2}+k_{r i}^{2}-k_{r o}^{2}=0 .
\end{aligned}
$$

The surface impedance as function of frequency (or $k$ ) at the inner ferrite boundary follows directly as 


$$
\Re=-j Z_{0} \frac{k_{r i}}{k} \frac{J_{0}\left(k_{r i} a\right)}{J_{1}\left(k_{r i} a\right)}=-j Z_{0} \frac{k_{r o}}{\varepsilon_{S} k} \frac{F_{0}\left(k_{r o} a\right)}{F_{1}\left(k_{r o} a\right)}
$$

For the typical case of thin cylinders, one finds

$$
\mathfrak{R} \approx j Z_{0} \frac{k_{r o}^{2}}{\varepsilon_{S} k}(b-a)
$$

and furthermore in the extreme relativistic case of $k=k_{z}$

$$
\Re \sim j Z_{0} \frac{\varepsilon_{S} \mu-1}{\varepsilon_{S}} k(b-a)
$$

Cavity resonances with $E_{r}=0$ on the end plates are found by imposing appropriate values for $k_{z}$, such as $k_{z}=0$ for the TM010 modes yielding $k=k_{r i}$ and $k_{r o}^{2}=\varepsilon_{S} \mu k_{r i}^{2}$. The eigenvalue solution is here reduced to the single impedance matching condition in $k_{r i}$

$$
\varepsilon_{S} J_{0}\left(k_{r i} a\right) F_{1}\left(k_{r i} a \sqrt{\varepsilon_{S} \mu}\right)-J_{1}\left(k_{r i} a\right) F_{0}\left(k_{r i} a \sqrt{\varepsilon_{S} \mu}\right)=0
$$

which provides the observable resonance frequency and the quality factor from

$$
f_{O}=\frac{C}{2 \pi} \operatorname{Re} k_{r i} \text { and } Q=\frac{\operatorname{Re} k_{r i}}{2 \operatorname{Im} k_{r i}}
$$

The analytical expressions were used in the Mathematica program with the ferrite permeability, $\mu=4.7(1-\mathrm{j} 2.55)$, to find the TM010 resonance frequency and quality factor in the VS cavity with $b=12.5$ and $a=12.1825 \mathrm{~cm}$. The "analytical" surface resistance is $R_{S}=167 \Omega$ is in reasonable agreement with the perturbation result, $R_{S}=133 \Omega$, from the geometry factor $G=453 \Omega$ and the $Q$ value. The "analytical" result, $f=731 \mathrm{MHz}$ and quality factor $Q=1.37$, is in good agreement with the MWS values of $f=719 \mathrm{MHz}$ and $Q=1.29$ for a solid cylinder. 